\title{
ICD-11: Impact on Traditional Chinese Medicine and World Healthcare Systems
}

\author{
Wai Ching Lam ${ }^{1} \cdot$ Aiping Lyu ${ }^{1} \cdot$ Zhaoxiang Bian $^{1}(\mathbb{C}$
}

Published online: 26 July 2019

(c) Springer Nature Switzerland AG 2019

\begin{abstract}
World Health Organization (WHO) member states adopted the eleventh revision of the International Statistical Classification of Diseases and Related Health Problems (ICD-11) on 25 May 2019. Historically, traditional Chinese medicine (TCM) was excluded from the ICD system. Including TCM in the ICD-11 is not only a landmark for the ICD but also a milestone for TCM: it enables the statistical data to cover information beyond Western medicine, thus reflecting the true situation of healthcare systems among member states of the WHO. This initiative is in line with the WHO Traditional Medicine Strategy (2014-2023), demonstrating that the WHO appreciates the past contribution of TCM to healthcare worldwide, and that it is responding to the current needs of its member states. Further, this action will have long-term impacts on TCM. It is a critical step not only because TCM may take this chance to be developed further among WHO member states, but also because TCM should benefit from improved service levels, education, research, and regulation. In addition, this initiative contributes to the progressive reform of the world healthcare system, from global extension of TCM to the integration of TCM with multiple Western medicine-based disciplines. People all over the world will benefit.
\end{abstract}

\section{Key Points}

Including traditional Chinese medicine (TCM) in the International Statistical Classification of Diseases and Related Health Problems (ICD) is a landmark for both TCM and ICD.

With this move, the World Health Organization (WHO) acknowledges the value of TCM, responds to member state needs, and advances the integration of TCM with Western medicine.

This initiative will stimulate further research, education, adoption, and recognition of TCM worldwide.

Zhaoxiang Bian

bzxiang@hkbu.edu.hk

1 School of Chinese Medicine, Hong Kong Baptist University, Hong Kong, SAR, China

\section{Introduction}

On 25 May 2019, World Health Organization (WHO) member states meeting at the World Health Assembly (WHA) adopted the eleventh revision of the International Statistical Classification of Diseases and Related Health Problems (ICD-11), developed by the WHO, which will come into effect on 1 January 2022 [1]. Compared with the tenth revision of the ICD (ICD-10), in addition to new concepts, content models, coding schemes, and terminology, ICD-11 includes a supplementary Chapter 26 for optional use that refers to 150 disorders and 196 patterns which originated from traditional Chinese medicine (TCM) and are commonly used in China, Japan, Korea, and elsewhere around the world [1]. This is the first time that TCM is included in the ICD system. Including the TCM into the ICD coding system, which was originally developed for Western medicine (WM), has not been easy, because the definition of disorders and patterns depends on, and arises from, classic concepts that are outside the Western medical paradigm [2]. The purposes of the ICD-11 are very clear. As stated in the 2016 strategy for the Joint Linearization for Mortality and Morbidity Statistics (JLMMS) of the ICD, this revision focused on developing (1) a classification of contents that meets the needs of the member states; (2) a sustainable statistic model 
beyond 2018; and (3) improved health information implementation beyond 2018 in both high- and low-resource settings [3]. This historic work of ICD-11 on traditional medicines is in line with the WHO Traditional Medicine Strategy (2014-2023), which encourages member states to regulate, promote research in, and integrate traditional medicine practices into their healthcare systems [4]. Including TCM into ICD-11 not only recognizes TCM for its past contribution to world healthcare, but also acknowledges, and attempts to serve, the current needs of the people for TCM.

\section{Historic Work}

\subsection{Landmark for the International Statistical Classification of Diseases and Related Health Problems (ICD)}

Chapter 26 of ICD-11 is a milestone for the ICD coding system, the development of which has been a long process. In 1893, with the aim of formulating a system for classifying causes of death, the first International List of Causes of Death was adopted by the International Statistical Institute, which serves as the start of the ICD coding system. Currently, the ICD contains around 55,000 unique codes for injuries, diseases, and causes of death. It works as a common language that allows health professionals in WM systems to share health information across the globe. Thus, it is a source of health statistics and the foundation for identifying health/disease trends worldwide. Although millions of people now use TCM, it has never previously been included in the ICD system. By now including TCM in the ICD system, the WHO officially acknowledges the value and impact of TCM in the management of disease and health-related problems for its member states. This revision of the ICD will make ICD statistical data more complete and more accurate.

\subsection{Landmark for Traditional Chinese Medicine (TCM)}

TCM and other traditional medicines originating from TCM, whose modalities range from acupuncture to herbal formulas and patent Chinese herbal medicine, Tai Qi, Chinese medicine massage, and bone setting, has been used in East Asia for more than 3000 years. Currently, it is widely used on almost every continent. To be included into the ICD system is a milestone for TCM, indicating that the past contributions to healthcare of TCM have been recognized, and the WHO is aware of current TCM needs in its member states. WM dominates the existing healthcare systems in many countries. However, experience shows that including TCM in a healthcare protocol improves outcomes for patients [5]. For example, TCM can provide good support to patients who suffer from some conditions, particularly those that are chronic and degenerative, e.g., irritable bowel syndrome [6] and low back pain [7]. Meanwhile, WM can treat the acute conditions that TCM cannot, e.g., myocardial infarction and cerebral hemorrhage. The two systems are truly complementary. Documented evidence has shown the effective integration of TCM and $\mathrm{WM}$ in treating pandemic viral diseases such as severe acute respiratory syndrome [8], influenza A virus subtype H1N1 [9], HIV and AIDS [10], and hand, foot and mouth disease [11], and this has attracted attention worldwide. Professor Tu Youyou won the 2011 Lasker Award in clinical medicine and the 2015 Nobel Prize in Physiology or Medicine for discovering artemisinin, an extract of a TCM herb Artemisia annua L., used to treat malaria. In addition, Dr. Wang Zhenyi and Dr. Chen Zhu were awarded the Seventh Annual Szent-Gyorgyi Prize for progress in cancer research for combining the WM all-trans retinoic acid and the TCM compound arsenic trioxide to treat acute promyelocytic leukemia [12].

TCM is becoming more and more widely used throughout the world. The WHO Global Atlas of Traditional, Complementary and Alternative Medicine shows that the majority of the world's population is using some form of traditional medicine on a regular basis, most often herbal medicines [13]. Currently, TCM is used in 183 countries and regions around the world. According to the WHO, 103 member states have approved the practice of acupuncture and moxibustion, 29 have enacted special statutes on traditional medicine, and 18 have included acupuncture and moxibustion treatment in their medical insurance provisions [12]. In 2016 in China, there were 3966 TCM hospitals across the country, 452,000 TCM practitioners, 42,528 TCM clinics, 910 million TCM outpatient visits, and $26,915,000$ inpatient treatments [12]. The export value of Chinese medicines amounted to \$US3.72 billion in 2015 [12]. In the USA, TCM treatment is being provided in conventional main stream clinics, such as the Cleveland Clinic [14], Dana-Farber Cancer Institute [15], Memorial Sloan Kettering Cancer Center [16], and Mayo Clinic [17]. As of January 2018, the number of licensed acupuncturists in the USA had increased by $257 \%$ compared with 1998 , reaching about 38,000 [18]. In Europe, many countries such as Germany, the UK, and France have set up TCM clinics. In Germany, the TCM Clinic Bad Kötzting has provided TCM outpatient and inpatient services since 1991 [19]. Most importantly, around 30 countries and regions now provide TCM professional or degree training [12]. The increases in TCM service and education within these countries reflects patients' need for and confidence in TCM, as well as the deficiencies of WM. Therefore, including TCM in ICD-11 is not just an appreciation of TCM's past contribution to healthcare systems worldwide, but also a response from the 
WHO to reflect the actual healthcare situation of member states with regards to TCM.

\section{Potential Impact on TCM}

Chapter 26 of ICD-11 refers to disorders and patterns as defined in TCM conceptual terms. It specifies recording epidemiological data about TCM practice from four aspects: (1) symptomology; (2) TCM etiology; (3) course and outcome; and (4) treatment response. This coding system will be a standard reference that all practitioners can use to help diagnose diseases and assess their possible causes during their practice. The implementation of this coding system (although optional) will affect, to some extent, how practitioners make diagnoses, how insurance companies determine coverage, and how government officers interpret healthcare statistical data. This will influence the service level, research, education, and regulation of TCM.

\subsection{Service}

ICD-11 is consistent with the WHO's Traditional Medicine Strategy (2014-2023) [4]. In order to be fit for statistical purposes, the WHO-recommended list of patterns and conditions will be used if member states choose to report TCM data; thus, the TCM data from different WHO member states would be comparable. For this purpose, the existing TCM service level should be improved, with at least standardization of the practice of TCM among different regions and countries, starting with the application of terminology. Further, the application of this recommended list will also impact on TCM service extension, because it opens a door for those member states who have exposure to TCM to promote its safe and effective use. Thus, ICD-11 also allows member states who have no experience in TCM to try it.

\subsection{Research}

ICD-11 is expected to trigger more research about TCM, at least regarding the four aspects discussed in Sects. 3.2.1-3.2.4.

\subsubsection{Standardization of Terminology}

Chapter 26 is a comprehensive list of medical conditions as described in TCM terms. The content and scope of each condition and pattern listed are based on the consensus of many meetings of the ICD revision steering committee [2]. However, establishing the list is only the first step. The terminology relating to the symptoms, etiology, course, outcome assessment criteria, and treatment response must be standardized, with clear descriptions. This is not a trivial exercise.
The symptomology refers to signs and symptoms as determined by traditional medicine diagnostic methods, including inspections such as tongue examination, history taking (inquiry), examination by listening and smelling, palpation such as pulse taking, and abdominal examination-most of which can be found in both TCM and WM but serving for entirely different diagnostic purposes. Some signs and symptoms, especially for unique findings by TCM, should be standardized. For example, the definition of tongue examination, such as tongue color, tongue form, tongue fur, etc., and its link to the diagnosis should be further studied and standardized. With standardization of these items, researchers will be able to gather, compare, and analyze the real-world TCM evidence from a variety of sources.

\subsubsection{Response Assessment}

Criticism continues about the efficacy and safety of TCM [20]. In fact, its efficacy is why TCM has been used for millennia. For any medicine or medical system, whether traditional or not, safety and efficacy will determine its service population. In the current evidence-based medicine era, including TCM in the ICD-11 will further trigger more rigorous research about its efficacy and safety in certain diseases and conditions. Although the last five decades have witnessed progress in TCM clinical research, the quality of this research, in terms of protocol design, implementation, data dissemination, and reporting, needs significant improvement [21]. Recently, some high-quality clinical research and clinical methodological works have been reported, reflecting the efforts of TCM researchers in this area [22, 23]. ICD-11, representing a new start for TCM, will trigger more response assessment to answer the question of whether TCM is helpful or harmful for certain conditions, and how it happens.

\subsubsection{Clinical Pathway and Practice Guideline}

Chapter 26 of ICD-11 will impact the development of the clinical pathways and practice guidelines for TCM, some of which have been proposed and published [21]. There is an urgency to systematically review these clinical pathways and practice guidelines because of quality issues [24]. ICD-11 provides a chance, and also puts on the pressure, to establish the clinical practice guideline and clinical pathway, based on the documented literature, especially evidence from rigorous clinical trials, thus securing standardization of the application of TCM processes. Evidence has shown that clinical pathway implementation can reduce the variability in clinical practice and improve the patients' outcomes, therefore ensuring the quality of the healthcare [25]. Furthermore, these clinical pathways and practice guidelines need to be promoted at the practice level among the WHO member 
states, where appropriate, thus maintaining the quality level of the clinical practice.

\subsubsection{TCM Drug Development}

Chapter 26 of ICD-11 could serve as a tool for pharmaceutical companies to acquire global standardized TCM data, to assess the market needs among different countries, and thereby determine the best development and marketing strategies. At the same time, companies producing TCM drugs need to improve the quality of their products in order to meet the regional and international standards for global marketing. It could be expected that preclinical, clinical, and postmarket study of TCM products will be significantly influenced by ICD- 11 .

\subsection{Education}

Including TCM in ICD-11 may expand its usage among WHO member states and thus more TCM practitioners will be needed to provide the service to the public. Also, WM doctors and related healthcare professionals may need to understand the concept and impact of TCM on their patients, who may also receive TCM treatment. Further, with potential increasing application of TCM, patients, their family members, and the public should know more about it. These potential needs will drive education changes, at least relating to three aspects: TCM professional degree education, TCM public education, and WM and other healthcare-related degree education. For TCM professional degree education, a TCM curriculum framework, especially regarding the subjects studied, practicum requirements, and licensing testing, should be set up, regionally or internationally, in order to maintain the quality standard. For WM practitioners and other healthcare professionals, there is a need for training in TCM, at the very least to understand the concept of TCM and its potential effects on their patients, especially when patients are receiving both TCM and WM treatment together. Three existing approaches to TCM training for WM professionals could serve as a reference: continuing education on TCM for WM practitioners, adding TCM concepts into the WM professional degree education, and an integrative degree curriculum with TCM and WM together [26]. To educate the public, governments, universities, the TCM industry, non-profit organizations, etc., can work together to promote knowledge of TCM at different levels. Joint efforts covering different aspects may meet the service needs of TCM for both different regions and worldwide.

\subsection{Regulation}

Regulations should be put in place to protect public health and maintain the professional standards of the Chinese medicine industry under the Chapter 26 of ICD-11. For this purpose, a regulation system for education providers, registration of Chinese medicine practitioners, registration of Chinese medicine products, and licensing of Chinese medicine traders, etc., will be needed. For the WHO member states with existing regulations, it would be beneficial to enhance their systems, and thus embrace the changes in ICD-11. For those member states without such a regulation system, it is better to proactively set up a regulation system. These regulations will finally protect patients' rights, and facilitate the development of traditional medicine.

\section{Potential Impact on World Healthcare}

Chapter 26 of ICD-11 will support member states to develop proactive policies and action plans relating to TCM. This action will progressively change the components of the world healthcare system and its pattern of service.

\subsection{TCM Extension}

As the WHO recognizes the global use of TCM as primary healthcare [6], implementation of ICD-11 will give TCM a standardized system of alphanumeric codes throughout the world for epidemiology and research, and for use in citations by the public, academic professionals, healthcare institutions, government departments, pharmaceutical industries, and insurance companies. The implementation of ICD-11 on 1 January 2022 will further promote the extension of TCM into the WHO member states, thus creating the potential for them to develop TCM services as a new element in their healthcare systems.

\subsection{Integration with the Existing Healthcare System}

ICD-11 will progressively transform the existing healthcare system, from a WM-dominant system to an integrative WM and TCM healthcare system. The rationale behind integration is to include the best practices of both conventional and complementary therapies, uniting these practices into an integrative approach, especially when positive evidence about the efficacy and safety of TCM for certain conditions and diseases is documented. The evidence will progressively change the WM-dominant practice guidelines of certain diseases [27], thus reframing the landscape of the healthcare system in the long run.

\section{Conclusions}

Chapter 26 of ICD-11 is a milestone for both the ICD and TCM. It enables the ICD system to cover more information beyond WM, and thus the statistical data can reflect the true 
situation of healthcare in member states of the WHO. This action will have a long-term impact on TCM itself, from the service level, education, and research to regulation. At the same time, this initiative will progressively reform healthcare systems worldwide.

\section{Compliance with Ethical Standards}

Funding The authors declare no funding support received in relation to this article.

Conflict of interest Wai Ching Lam, Aiping Lyu and Zhaoxiang Bian declare no conflicts of interests that relate to this article.

\section{References}

1. World Health Organization. World Health Assembly update, 25 May 2019. 2019. https://www.who.int/news-room/detail/25-052019-world-health-assembly-update. Accessed 27 May 2019.

2. Nature News. Why Chinese medicine is heading for clinics around the world. 2018. https://www.nature.com/articles/d41586-01806782-7. Accessed 27 May 2019.

3. World Health Organization. ICD-11 Joint Linearization for Mortality and Morbidity Statistics: project plan 2015-2018. 2019. https://www.who.int/classifications/icd/revision/icdprojectplan2 015to2018.pdf. Accessed 27 May 2019.

4. World Health Organization traditional medicine strategy:2014-2023. 2019. https://www.who.int/medicines/publicatio ns/traditional/trm_strategy14_23/en/. Accessed 27 May 2019.

5. Maizes V, Rakel D, Niemiec C. Integrative medicine and patientcentered care. Explore. 2009;5(5):277-89.

6. Bi Z, Zheng Y, Yuan J, Bian Z. The efficacy and potential mechanisms of Chinese herbal medicine on irritable bowel syndrome. Curr Pharm Des. 2017;23(34):5163-72.

7. Yuan QL, Guo TM, Liu L, Sun F, Zhang YG. Traditional Chinese medicine for neck pain and low back pain: a systematic review and meta-analysis. PLoS One. 2015;10(2):e0117146.

8. Liu X, Zhang M, He L, Li Y. Chinese herbs combined with Western medicine for severe acute respiratory syndrome (SARS). Cochrane Database Syst Rev. 2012;10:CD004882.

9. Li JH, Wang RQ, Guo WJ, Li JS. Efficacy and safety of traditional Chinese medicine for the treatment of influenza A (H1N1): a meta-analysis. J Chin Med Assoc. 2016;79(5):281-91.

10. Wang J, Zou W. Practices, challenges, and opportunities: HIV/ AIDS treatment with traditional Chinese medicine in China. Front Med. 2011;5(2):123-6.

11. Li XH, Li SJ, Xu Y, Wei D, Shi QS, Zhu QX, et al. Effect of integrated Chinese and Western medicine therapy on severe hand, foot and mouth disease: a prospective, randomized, controlled trial. Chin J Integr Med. 2017;23(12):887-92.

12. The State Council Information Office of the People's Republic of China. Traditional Chinese medicine in China. 2016. http://engli sh.gov.cn/archive/white_paper/2016/12/06/content_2814755093 33700.htm. Accessed 27 May 2019.

13. Ong CK, Bodeker G, Grundy C, Burford G, Shein K. WHO global atlas of traditional, complementary and alternative medicine.
World Health Organization, Kobe. 2005. https://apps.who.int/ iris/handle/10665/43108?locale-attribute $=$ en $\&$. Accessed 27 May 2019.

14. Cleveland Clinic. Chinese herbal therapy. 2019. https://my.cleve landclinic.org/departments/wellness/integrative/treatments-servi ces/chinese-herbal-therapy. Accessed 27 May 2019.

15. Dana-Farber Cancer Institute. Zakim Center clinical services. 2019. https://www.dana-farber.org/for-patients-and-families/ care-and-treatment/support-services-and-amenities/zakim-cente r-for-integrative-therapies/clinical-services/. Accessed 27 May 2019.

16. Memorial Sloan Kettering Cancer Center. Integrative medicine. 2019. https://www.mskcc.org/cancer-care/diagnosis-treatment/ symptom-management/integrative-medicine. Accessed 27 May 2019.

17. Mayo Clinic. Acupuncture. 2018. https://www.mayoclinic.org/ tests-procedures/acupuncture/about/pac-20392763. Accessed 27 May 2019.

18. Arthur YF, Steven HS, Sarah FA, Amy M. Distribution of licensed acupuncturists and educational institutions in the United States at the start of 2018. Complement Ther Med. 2018;41:295-301.

19. TCM Clinic Bad Kötzting. TCM Clinic. 2019. http://www.tcmklinik-koetzting.de/tcmen/tcm-klinik/index.php. Accessed 27 May 2019.

20. The world health organization's decision about traditional Chinese medicine could be backfire [editorial]. Nature. 2019; 570(7759):5. https://doi.org/10.1038/d41586-019-01726-1.

21. Chen YL, Zhao C, Zhang L, Li B, Wu CH, Mu W, et al. Toward evidence based Chinese medicine: status quo, opportunities and challenges. Chin J Integr Med. 2018;24(3):163-70.

22. Liu Z, Liu Y, Xu H, He L, Chen Y, Fu L, et al. Effect of electroacupuncture on urinary leakage among women with stress urinary incontinence: a randomized clinical trial. JAMA. 2017;317(24):2493-501. https://doi.org/10.1001/jama.2017.7220.

23. Zhong LLD, Cheng CW, Kun W, Dai L, Hu DD, Ning ZW, et al. Efficacy of MaZiRenWan, a Chinese herbal medicine, in patients with Functional constipation in a randomized controlled trial. Clin Gastroenterol Hepatol. 2019;17(7):1303-1310.e18. https:// doi.org/10.1016/j.cgh.2018.04.005.

24. Yun X, YaoLong C, Zhao Z, Qi Z, Yang Yang W, RunShen X, et al. Using the RIGHT Statement to evaluate the reporting quality of clinical practice guidelines in traditional Chinese medicine. PLoS One. 2018;13(11):e0207580. https://doi.org/10.1371/journ al.pone. 0207580 .

25. Rotter T, Kinsman L, James E, Machotta A, Gothe H, Willis J, et al. Clinical pathways: effects on professional practice, patient outcomes, length of stay and hospital costs. Cochrane Database Syst Rev. 2010. https://doi.org/10.1002/14651858.CD006632. pub2.

26. Zhu W, Wang XM. Current situation and development strategy of education of integrated traditional Chinese and Western medicine. Tianjin J Tradit Chin Med. 2006;23(5):386-7.

27. Department of Veterans Affairs/Department of Defense. VA/DoD clinical practice guideline for management of asthma in children and adults. VA/DoD evidence-based practice. 2009. https:// www.healthquality.va.gov/guidelines/CD/asthma/ast_2_full.pdf. Accessed 27 May 2019. 